\title{
How to be fairer
}

\section{Conrad Heilmann ${ }^{1}$. Stefan Wintein ${ }^{2}$}

Received: 6 March 2015 / Accepted: 5 November 2015 / Published online: 12 December 2015 (C) The Author(s) 2015. This article is published with open access at Springerlink.com

\begin{abstract}
We confront the philosophical literature on fair division problems with axiomatic and game-theoretic work in economics. Firstly, we show that the proportionality method advocated in Curtis (in Analysis 74:417-57, 2014) is not implied by a general principle of fairness, and that the proportional rule cannot be explicated axiomatically from that very principle. Secondly, we suggest that Broome's (in Proc Aristot Soc 91:87-101, 1990) notion of claims is too restrictive and that game-theoretic approaches can rectify this shortcoming. More generally, we argue that axiomatic and game-theoretic work in economics is an indispensable ingredient of any theorizing about fair division problems and allocative justice.
\end{abstract}

Keywords Cooperative game theory · Fairness - Bankruptcy · Fair division • Proportionality

\section{Introduction}

There is not enough cake: somebody took most of it and now only five slices are left. Luc wants three of them, Cas four, and Sef wants two slices. There is not enough money: Alice has only 20 Euros left. She had borrowed 8 Euros from Alex, 11 from Ben, and 5 from Sarah. Many situations in life are like that. When in charge of allocating a limited

Conrad Heilmann

heilmann@fwb.eur.nl

Stefan Wintein

stefanwintein@gmail.com

1 Erasmus Institute for Philosophy and Economics (EIPE), Faculty of Philosophy, Erasmus University Rotterdam, Rotterdam, The Netherlands

2 Faculty of Philosophy, Erasmus University Rotterdam, Rotterdam, The Netherlands 
amount of an available good, a fair division problem arises: how do you ensure that you treat everyone fairly?

Perhaps the best known theory of fairness that applies to such situations is that of Broome (1990). Its core feature is that fairness is the proportional satisfaction of claims, which are a certain type of reasons, owed to the agent, as to why an agent should have the good. Broome (1990, p. 95) understands 'proportional satisfaction' in the following way: 'I do not mean 'proportion' to be taken too precisely. But I do mean that equal claims require equal satisfaction, that stronger claims require more satisfaction than weaker ones, and also - very importantly - that weaker claims require some satisfaction. Weaker claims may not be simply overridden by stronger ones.' Broome is thus quite non-committal about the notion of proportionality, and his theory does not offer an actual method that describes 'how to be fair'. In response to this, Curtis (2014) has provided a new theory of fairness that spells out what he maintains Broome's (1990) theory has left out.

The core feature of Curtis' theory is what he calls the proportionality method, a protocol to be used by the allocating agent to divide the good proportionally. Yet, like all philosophical investigations into fair division problems of the type mentioned above that we are aware of, Curtis develops his theory in isolation from axiomatic and cooperative game-theoretic approaches to fairness in economics, whose main focus is precisely to compare different methods of dividing a limited amount of an available good (e.g. Herrero and Villar 2001; Thomson 2003; O’Neill 1982; Curiel et al. 1988). In this paper, we analyze the philosophical literature on fair division problems from the viewpoint of these approaches. We argue that the proportionality method does not follow from the general principle of fairness from which Curtis (2014) starts out. We also find that several of the 'Broomean' assumptions that Curtis makes, which have so far been the focal point of the recent philosophical literature on fairness, are equally implausible. In particular, Broome's restricted notion of what a 'claim' in a fair division problem is, already criticized by Hooker (2005), Tomlin (2012), and Kirkpatrick and Eastwood (2015), renders his theory of fairness inapplicable in many important contexts. The economic literature, by contrast, offers a range of tools in which such assumptions can be dropped, and thus offers a fruitful environment for the study of fairness.

We proceed as follows. In Sect. 2, we show that Curtis' (2014) proportionality method does not follow from the general principle of fairness that he adopts. First we show that there are several fair division rules, studied in the economic literature, that are consistent with it. We then invoke the axiomatic literature on fairness to investigate whether there is an explication of the fairness principle that rescues Curtis' claim. We will consider a number of properties that jointly may serve as an explication of Curtis' principle of fairness and demonstrate that these do not jointly imply the proportionality method. Finally, we argue that no explication of Curtis' principle of fairness will do so, by invoking Thomson's (2012) distinction between punctual and relational properties. In Sect. 3, we reflect briefly on the significance and usefulness of the axiomatic approach for philosophical work on fair division problems. In Sect. 4, we consider situations in which claims are not only less idealized than Broomean (1990) claims, but rather implicit and intertwined with other aspects of the context at hand. Solution concepts from cooperative game theory supply the necessary tools to investigate fairness in such situations. Moreover, we demonstrate that there are cases in 
which cooperative game theory applies that cannot even be reduced to situations with Broomean claims. We also point out a drawback of the proportional rule: unlike many other division rules, it is not robust, as it cannot be characterized both axiomatically and game theoretically. In Sect. 5, we reflect on the significance of our findings for theorising about fair division problems and allocative justice more generally. In Sect. 6, we conclude.

\section{Curtis on fairness}

\subsection{FC does not imply $P$}

\section{Consider the following situation.}

Bankruptcy. Ari, Betty, and Claire have, respectively, deposited 800, 400 and 200 Euros on their savings account with the SAFE bank. However, SAFE goes bankrupt and its liquidation value of 900 (the 'estate') has to be divided amongst Ari, Betty, and Claire. Together, the money on their accounts adds up to 1400, and so a problem arises. How should we, in order to be fair, divide the estate?

Recently, Curtis (2014) has provided an important new theory of fairness that applies to such problems. ${ }^{1}$ Curtis advocates his theory as spelling out what he maintains Broome's (1990) well-known theory of fairness has left out: an actual method that describes 'how to be fair'. Curtis (2014, p. 47) remarks that, to the best of his knowledge, 'neither Broome nor anyone else had laid down a theory of precisely what one must do in order to be fair'. Yet, fair division problems like Bankruptcy have been studied extensively, both axiomatically (see Herrero and Villar 2001 and Thomson 2003 for overviews) and from the perspective of cooperative game theory (see e.g. O'Neill 1982 or Curiel et al. 1988). There is a long history to the study of such problems. For instance, the Talmud discusses a division rule, which has been studied formally by Aumann and Maschler (1985).

Classical examples of division rules are the rule of 'constrained equalized awards' (CEA), the 'proportional rule' (P), and the rule of 'constrained equalized losses' (CEL). There are many more rules in the axiomatic and game-theoretic literature, with some of the most important ones being introduced in this paper as we go along. Let us for now focus on the three rules just mentioned and apply them to Bankruptcy. The CEA rule equalizes the awards as much as possible, under the constraint that no agent can receive more than his or her claim. ${ }^{2}$ In Bankruptcy, the estate is 900 and so allotting

\footnotetext{
1 Curtis' theory applies both to cases in which the allocated good is infinitely divisible and to cases in which it is not. The proportionality method is the cornerstone of his treatment of both cases however, and Curtis' treatment of the non-infinitely divisible case is an extension of his treatment of the infinitely divisible one. For our purposes, it suffices to restrict ourselves to the simpler case in which the good is infinitely divisible. However, it needs to be said that fair division problems with a non-infinitely divisible good are less well-studied in the economic literature that we consider here than those in which the good is infinitely divisible.

2 We will make the notion of 'claim' more precise in the next sections. For the moment, we just take the amounts of Euros in Bankruptcy at face value. In Sect. 4.1, we will deal with a potential rejoinder from Curtis that is based on a specific notion of what a claim is.
} 
Table 1 Division rules and their recommended awards for Bankruptcy

\begin{tabular}{llllr}
\hline Agents & Claims & CEA & P & CEL \\
\hline Ari & 800 & 350 & $514 . \overline{3}$ & $633 . \overline{3}$ \\
Betty & 400 & 350 & $257 . \overline{1}$ & $233 . \overline{3}$ \\
Claire & 200 & 200 & $128 . \overline{6}$ & $33 . \overline{3}$ \\
\hline
\end{tabular}

300 to each of the agents equalizes the awards. However, doing so would give Claire more than her claim of 200. So Claire gets 200 and the remaining 700 is shared equally between Ari and Betty. Now none of the three agents gets more than his or her claim, and the resulting allocation is the one prescribed by the CEA rule, as indicated in Table 1.

Further, $\mathrm{P}$ divides the estate in proportion to the size of the claims of the respective agents. Finally, the CEL rule equalizes the losses that the agents suffer with respect to their claims under the constraint that no agent can loose more than his or her claim. Suppose that we ('temporarily') allot Ari, Betty and Claire the totality of their claims. This would result in an infeasible allocation, as we would distribute 1,400 whereas the estate is only 900. To reach a feasible allocation, the CEL rule proposes that the agents equally share the aggregate loss of 1,400-900 $=500$. Hence, the CEL rule proposes that each agent loses $166 . \overline{7}$ relative to his or her claim, resulting in the allocation that is depicted in Table 1 above.

For claims problems like Bankruptcy, Curtis (2014) endorses the proportional rule P. Indeed, P is the cornerstone of his theory of fairness. What is more, he motivates adopting it by claiming that the proportional rule is entailed by a general principle of fairness that he adopts:

The Fairness Claim (FC). In order to be fair an allocating agent must (i) do as much as she can to satisfy the claim of each receiving agent to as great a degree as possible whilst ensuring that (ii) each claim is treated equally (Curtis 2014, p. 49).

Now FC is fairly general and open to interpretation. But Curtis does not provide any. Rather, he simply states that the allocating agent 'divides ...proportionally is demanded by part (ii) [of FC]' and that 'it is also clear that FC tells [the allocating agent] to follow method P' (Curtis 2014, p. 49). In a nutshell, Curtis claims that FC implies P.

We submit that the content of FC is too vague to single out $\mathrm{P}$ as the only division rule that is consistent with it. For one thing, all the rules we introduced in the above example realise FC (i) and of all of them it can reasonably be said that they treat each claim equally. To be sure, $\mathrm{P}$ does not seem to be in conflict with $\mathrm{FC}$, and it can be argued that $\mathrm{P}$ is a plausible way to realise FC. But to the extent to which that can be argued, the same can be said about the CEA and CEL rules. FC is simply open to many interpretations and does not lead to one division rule. In other words: FC does not imply $\mathrm{P}$.

We have already mentioned that many division rules have been developed in the axiomatic literature. In Sect. 2.2, we will employ this literature to propose an explication of FC. As we will see, our FC explication does not imply P. In Sect. 2.3 we go on to argue that no plausible explication of FC in the axiomatic approach can imply $\mathrm{P}$. 
The following two sections, as well as Sects. 4.2 and 4.3, are more technical than the rest of the article, which we indicate by putting a star next to their title. While the general message of the article is conveyed by the non-starred sections, the starred sections demonstrate the fruitfulness of the axiomatic and game-theoretic methods for theorizing about fair divisions in detail.

\subsection{Explicating FC: using the axiomatic approach ${ }^{\star}$}

The axiomatic approach analyzes cases like Bankruptcy as fair division problems with a specific structure, which is that of a so-called claims problem.

Definition 1 A claims problem $\mathcal{C}:=(E, N, c)$ consists of an estate $E>0$, a set of agents $N=\{1, \ldots, n\}$ and a claims vector $c \in \mathbb{R}_{+}^{N}$ specifying a claim $c_{i}$ for each agent $i$ such that $\sum_{i \in N} c_{i}>E$.

A multitude of division rules for claims problems have been proposed and studied in the literature.

Definition 2 A division rule $r$ is a function that maps each claims problem $(E, N, c)$ to an allocation vector $x \in \mathbb{R}_{+}^{N}$, with the property that $x_{i} \leq c_{i}$ for each agent $i$ : no agent receives more than his claim.

On the axiomatic approach, such rules are characterized in terms of logically independent properties which facilitates a fruitful comparison of (the fairness of) distinct division rules. One of the rules considered is the proportional one, about which Thomson remarks:

The best-known rule is the proportional rule, which chooses awards proportional to claims. Proportionality is often taken as the definition of fairness [...], but we will challenge this position and start from more elementary considerations. (Thomson 2003, p. 250).

Indeed, Curtis does not want to take proportionality as the definition of fairness, but wants to start from more elementary considerations that are encapsulated in FC. And so, the axiomatic approach seems to be highly relevant for Curtis' project.

Let us first illustrate the main tenets of the axiomatic approach by exploring the division rules already mentioned earlier. The CEA, P and CEL rule all have the Efficiency property as, in each claims problem, the rules allocate all of the available estate. Another property that is common to the three rules is that of Equal Treatment of Equals, which says that (in each claims problem) agents with the same claims receive the same amount. Efficiency and Equal Treatment of Equals are logically independent properties: a division rule may satisfy Efficiency without satisfying Equal Treatment of Equals, or vice versa. Further, Efficiency and Equal Treatment of Equals are shared by lots of division rules, and hence these properties do not (jointly) characterize a division rule, i.e. there is no unique division rule satisfying them. To characterize a division rule is to identify a rule as the only one that satisfies a certain set of (preferably logically independent) properties. 
The core business of the axiomatic approach is exactly to characterize division rules in this sense. Such characterizations facilitate a fruitful comparison of (the fairness of) distinct division rules. In this section, we will use the axiomatic approach to explicate FC.

Remember that Curtis (2014, p. 49) says, 'that [the allocating agent] must use all of the good is demanded by part (i) of FC'. In terms of the axiomatic approach, Curtis claims that FC (i) demands a division rule that satisfies Efficiency. With this, we have no qualms. However, it should be noted that at this point, Curtis' theory differs from that of Broome. According to Broome, fairness is a strictly comparative value that only requires the proportional satisfaction of claims: Broome denies that fairness requires Efficiency. Allotting 8 Euros to Ari, 4 Euros to Betty and 2 Euros to Claire in Bankruptcy is, according to Broome, just as fair as the division proposed by P. Various authors have objected to the Broomean thought that fairness is strictly comparative. For instance, Hooker (2005, p. 341) argues that, pace Broome, 'fairness requires the greatest possible satisfaction of claims'. Indeed, Curtis states that he departs from Broome regarding this point, for the same reasons given by Hooker. Thus, although it is clear that FC (i) expresses Efficiency, it is debatable whether fairness requires Efficiency, i.e. whether Efficiency is a fairness property. For the purpose of this paper, however, we will simply follow Curtis and Hooker and assume that Efficiency, which is expressed by FC (i), is a fairness property.

Which fairness properties - allegedly characterizing $\mathrm{P}$ in the presence of Efficiency_are expressed by FC (ii)? Recall that FC (ii) is fairly general and open to interpretation. But perhaps we can invoke the axiomatic approach to understand its content in a more precise way. To do so, we look for a characterization of $\mathrm{P}$ in terms of properties that best reflect the phrase 'to treat equal claims equally'. For this purpose, the following characterization of $\mathrm{P}$ looks attractive.

Theorem 1 The proportional rule P is the only division rule that satisfies the following logically independent properties: Efficiency, Equal Treatment of Equals, Self-Duality and Composition.

\section{Proof See Young (1988).}

Let us first explain these properties in turn. We already encountered Efficiency and Equal Treatment of Equals. Explaining Self-Duality requires a little more work. Given a claims problem $(E, N, c)$ and a division rule $r$ that satisfies Efficiency, there are two canonical manners in which $r$ can be exploited to divide the estate amongst the agents. First, by applying $r$ directly to the problem at hand. Second, by applying $r$ to the loss problem $\left(\sum_{i \in N} c_{i}-E, N, c\right)$ that is associated with $(E, N, c)$, and by diminishing each agent's claim with the loss that is allocated to him or her as such. Thus, we may exploit $r$ either to 'divide what there is' or to 'allocate what is missing'. The second manner to divide the estate may also be thought of as applying the dual rule $r^{\star}$ of $r$ to $(E, N, c)$ :

$$
r^{\star}(E, N, c)=c-r(L, N, c), \quad \text { with } L=\sum_{i \in N} c_{i}-E \text { the aggregate loss. }
$$


Indeed, each efficient division rule comes with a dual rule. Whereas CEA is the dual rule of CEL (and vice versa), the dual rule of $\mathrm{P}$ is $\mathrm{P}$ itself, which is to say that $\mathrm{P}$ satisfies Self-Duality. For self-dual rules, 'dividing what there is' is tantamount to 'allocating what is missing': such rules treat awards and losses in a symmetrical manner. When a division rule satisfies Composition, it doesn't matter whether we divide all of the estate $E$ at once or whether we divide, first, a part $E_{1}$ of the estate and, second, the remainder $E_{2}$ according to the outstanding claims after the first stage. More precisely, a division rule $r$ satisfies Composition just in case, for all $E_{1}, E_{2}>0$ such that $E_{1}+E_{2}=E$, we have that:

$$
r(E, N, c)=r\left(E_{1}, N, c\right)+r\left(E_{2}, N, c-r\left(E_{1}, N, c\right)\right)
$$

Theorem 1 thus tells us that Efficiency, Equal Treatment of Equals, Self-Duality and Composition jointly characterize P. How then, are these properties related to FC? As we already discussed, Efficiency is expressed by FC (i) and-although debatablewe assume that fairness requires Efficiency. Equal Treatment of Equals wears its interpretation on its sleeves: (agents with) equal claims should receive the same, i.e. they should be treated equally. As FC (ii) says that each claim should be treated equally, it follows from FC (ii) that, in particular, equal claims should be treated as such. Hence, Equal Treatment of Equals is implied by FC (ii). What about Self-Duality? Although Self-Duality is not, strictly speaking, implied by FC (ii), treating awards and losses in a symmetrical manner can, being charitable to Curtis, be understood as treating claims equally: whereas CEA and CEL are biased towards equalizing awards and losses respectively, and do not satisfy Self-Duality, $\mathrm{P}$ is free of these biases and treats each claim equally, no matter whether we allocate what is there or what is missing.

What about the last property on our list, Composition? Here, we feel that we run out of charity. Not only do we not see any relation between FC (ii) and Composition, we think that it is hard to see any relation between fairness and Composition at all. We concur with Herrero and Villar (2001), who classify Composition as a requirement that prevents an allocation to depend on the way in which a claims problem is subdivided into partial problems. Thus, although one might interpret Composition as a generally desirable property, it is hard to qualify it as a fairness property. Moreover, like Efficiency and Equal treatment of Equals, Composition is a property that is common to CEA, P and CEL. Hence, not only is the relation between Composition and fairness opaque, Composition does not, whereas Self-Duality does, allow us to distinguish CEA and CEL from P.

Efficiency, Equal Treatment of Equals and Self-Duality do not jointly characterize $\mathrm{P}$, which follows from Theorem 1 and which is illustrated more vividly by the fact that they are also satisfied by the Talmud rule (cf. Aumann and Maschler 1985), the Run to the Bank rule (cf. O'Neill 1982) and the Adjusted Proportional rule (cf. Curiel et al. 1988). ${ }^{3}$ We discuss the Adjusted Proportional (AP) rule in some detail here, as it will play a role in the remainder of the paper.

\footnotetext{
${ }^{3}$ For proofs that the Talmud, Run to the Bank and Adhusted Proportional rule satisfy Efficiency, Equal Treatment of Equals and Self-Duality see, respectively, Aumann and Maschler (1985), O'Neill (1982) and Curiel et al. (1988).
} 
The AP rule exploits the notion of the minimal right of an agent, which is that part of the estate that would be left when the claims of all other agents were fully satisfied. In Bankruptcy, completely satisfying the claims of Betty and Claire would leave $900-400-200=300$ for Ari, which is his minimal right. The minimal rights of Betty and Claire are 0 for each. The AP rule proposes to distribute minimal rights first. Doing so in Bankruptcy leaves us with an estate of $900-300=600$, which is called the remaining estate. Further, allotting Ari's minimal right reduces his original claim with 300 , resulting in a reduced claim of $800-300=500$ which, as this reduced claim does not exceed the remaining estate, is also his remaining claim $^{4}$. The remaining claims for Betty and Claire are thus equal to their original claims of, respectively, 400 and 200. The AP rule distributes minimal rights first and then divides the remaining estate according to the remaining claims, resulting in an allocation that allots $572 . \overline{7}$ to Ari, $218 . \overline{2}$ to Betty, and $109 . \overline{1}$ to Claire.

As the AP rule satisfies Efficiency, Equal Treatment of Equals and Self-Duality it cannot, in virtue of Theorem 1, satisfy Composition. However, as Composition is not even remotely connected to FC, Curtis cannot appeal to Composition to argue that $\mathrm{P}$ is preferable to AP according to his conception of fairness. But perhaps there are other properties - more in line with FC - that would allow Curtis to do so? Consider the property of Strict Order Preservation, which says that whenever a claims problem $\mathcal{C}$ is such that agent $i$ has a claim that is strictly greater than the claim of agent $j$, the amount allotted to $i$ should be strictly greater than the amount allotted to $j$. Strict Order Preservation seems to be not too far removed from the content of FC (ii) and certainly to be in its spirit: remember that Broome (1990, p. 95) writes that 'stronger claims require more satisfaction than weaker ones' which is tantamount to Strict Order Preservation. Moreover, P does satisfy Strict Order Preservation, whereas AP does not. The former is immediate from its definition, the latter is readily verified by considering a claims problem in which there are two agents that have unequal claims which both exceed the available estate. The following lemma, which will be exploited later on, attests that AP only violates Strict Order Preservation in claims problems that satisfy the aforementioned condition.

Lemma 1 Let $(E, N, c)$ be a claims problem and let $k, l \in N$ be such that $c_{k}<c_{l}$. Then, $A P_{k}(E, N, c)<A P_{l}(E, N, c)$ if and only if $c_{k}<E$.

Proof See the appendix.

We use Lemma 1 in the proof of the following proposition, where we present a division rule_called the Composite Proportional rule for reasons that will become apparentthat satisfies Efficiency, Equal Treatment of Equals, Self-Duality and Strict Order Preservation, but that does not coincide with $\mathrm{P}$.

Proposition 1 Efficiency, Equal Treatment of Equals, Self-Duality and Strict Order Preservation do not characterize $P$.

4 If the reduced claim exceeds the remaining estate, the remaining claim is set equal to the remaining estate. 
Proof With $L=\sum_{i \in N} c_{i}-E$ the aggregate loss, the Composite Proportional rule $\mathrm{CP}$ is defined as follows.

$$
C P(E, N, c)= \begin{cases}P(E, N, c) & \exists k, l \in N: c_{k}<c_{l} \text { and }\left(c_{k} \geq E \text { or } c_{k} \geq L\right) \\ A P(E, N, c) & \text { otherwise }\end{cases}
$$

That rule CP satisfies Efficiency and Equal Treatment of Equals follows immediately from the fact that $\mathrm{P}$ and AP do. To see that R satisfies Self-Duality observe that $\mathrm{CP}$ is defined in such a way that for each claims problem $(E, N, c)$ either CP applies $\mathrm{P}$ to both $(E, N, c)$ and $(L, N, c)$ or CP applies AP to both $(E, N, c)$ and $(L, N, c)$. And so, as both P and AP are Self-Dual, so is CP. To see that CP satisfies Strict Order Preservation, observe that it follows from Lemma 1 that $\mathrm{CP}$ applies $\mathrm{P}$ - which satisfies Strict Order Preservation - to a claims problem when applying the AP rule would result in a violation of Strict Order Preservation. Finally, observe that R proposes to apply AP to Bankruptcy and that P and AP do not coincide on Bankruptcy. Hence CP does not coincide with $P$.

Proposition 1 shows that the explication of FC in terms of Efficiency, Equal Treatment of Equals, Self-Duality and Strict Order Preservation does not imply P. We will now go on to argue that no (satisfactory) explication of FC can imply $\mathrm{P}$, since that is prevented by the type of properties that are referred to by FC.

\subsection{Limits to FC explications: relational properties ${ }^{\star}$}

How might Curtis react to Proposition 1? While we do not know that, the following reaction seems natural.

Reaction Proposition 1 crucially relies on your rule CP. However, rule CP is ad hoc, as it proposes to apply $\mathrm{P}$ or AP, depending on whether some cooked up formal condition obtains. A minimal requirement on a fair division rule is exactly that it is not ad hoc, or more positively, that it exhibits something like 'regularity' or 'predictability'. The ad hoc character of $\mathrm{CP}$ reveals that is not a fair division rule. $\mathrm{P}$ is vastly superior.

Indeed, CP has an 'ad hoc character' and we sympathise with the thought that this is incompatible with fairness. But then, we may ask, how can we find out whether a given rule is 'ad hoc' in this way? Here is a proposal. A division rule $r$ satisfies Estate Continuity ${ }^{5}$ just in case small changes in the estate do not lead to large changes in the allocation vector that is proposed by $r$. The ad hoc character of CP can be explained by its violation of Estate Continuity, and this violation can be illustrated as follows. Suppose that Ari, Betty, and Claire have claims of 800, 400, and 200 with respect to an estate of 400.1. Inspection reveals that rule CP proposes to apply AP to this claims problem, resulting in an allocation of $160 . \overline{1}$ for Ari. However, if the estate would be slightly lowered to 400 (leaving the claims as they are), CP proposes to apply $\mathrm{P}$, which

\footnotetext{
5 Formally, $r$ satisfies Estate Continuity just in case $r_{i}(E, N, c)$ is a continuous function in $E$, for each $i \in N$.
} 
results in an allocation of $228 . \overline{6}$ for Ari. Hence, a small change in the estate results in a large change in the awards vector, which shows that $\mathrm{CP}$ violates Estate Continuity.

By appealing to Estate Continuity, we may thus account for the ad hoc character of $\mathrm{CP}$ and, as $\mathrm{P}$ satisfies Estate Continuity, this property can be used to distinguish $\mathrm{P}$ from CP. Moreover, one may even go further and argue that Estate Continuity is a property that any fair division rule should have. One may, but Curtis cannot. Or so we will argue. It is not only hard to see how, intuitively, Estate Continuity is related to FC, we will also show that FC does not give us access to relational properties such as Estate Continuity; all that FC has access to are punctual properties.

The distinction between punctual and relational properties is due to Thomson (2012). To illustrate this distinction, we contrast the property of Strict Order Preservation, as discussed before, with that Strict Claim Monotonicity. When two claims problems $\mathcal{C}$ and $\mathcal{C}^{\prime}$ are exactly alike, except for the fact that there is exactly one agent, say $i$, who has a strictly larger claim in problem $\mathcal{C}^{\prime}$ than in $\mathcal{C}$, Strict Claim Monotonicity says that agent $i$ should receive strictly more in problem $\mathcal{C}^{\prime}$ than in problem $\mathcal{C}$. In some sense, both Strict Order Preservation and Strict Claim Monotonicity can be said to give substance to the thought that 'stronger claims require more satisfaction'. In contrast to Strict Order Preservation though, Strict Claim Monotonicity allows one to distinguish $\mathrm{P}$ from our $\mathrm{CP}$ rule, as the following proposition attests.

\section{Proposition 2 P does and CP does not satisfy Strict Claim Monotonicity.}

\section{Proof See Appendix.}

Now given that the substance of Strict Claim Monotonicity is not too far removed from that of Strict Order Preservation, and given that the latter property may legitimately serve in an FC explication, can't Curtis appeal to Strict Claim Monotonicity in order to distinguish $\mathrm{P}$ from CP? No he cannot, for although the substance of Strict Claim Monotonicity may not be too far removed from that of Strict Order Preservation, it is a property of a different type.

Strict Order Preservation is a punctual property. In order to satisfy a punctual property, a division rule has to respect a certain condition in every claims problem. Now a division rule satisfies Strict Order Preservation just in case, in every claims problem 'strictly higher claims receive strictly more satisfaction'. Hence, Strict Order Preservation is a punctual property. Strict Claim Monotonicity is a relational property. In order to satisfy a relational property, a division rule has to respect a certain condition pertaining to each tuple of claims problems that are related in a certain way. Now a division rule satisfies Strict Claim Monotonicity just in case, for every pair of claims problems $\mathcal{C}$ and $\mathcal{C}^{\prime}$ that are related as described above, agent $i$ receives strictly more in $\mathcal{C}^{\prime}$ than in $\mathcal{C}$. Hence, Strict Claim Monotonicity is a relational property.

FC can only be explicated by punctual properties as both FC (i) and FC (ii) specify what a fair allocating agent must do in every claims problem. In particular, FC(ii) specifies that in order to be fair, an allocating agent must "ensure that [in every claims problem] each claim is treated equally'. As FC can only express punctual properties, we take it that it is reasonable to require, of an explication of FC, that it only appeals to punctual properties: call this the FC Explication Requirement. And so, according to the FC Explication Requirement, no explication of FC can appeal to Strict Claim 
Monotonicity or Estate Continuity. Thus, although these properties allow one to separate $\mathrm{P}$ from $\mathrm{CP}$, Curtis cannot justify this separation by appealing to FC.

Just like Strict Order Preservation, Efficiency and Equal Treatment of Equals are clear instances of punctual properties. Moreover, these three properties are not only of the right type to serve in an explication of FC, but also their substance is closely related to that of FC. Hence, their occurrence in an FC explication is unproblematic. But what about Self-Duality? As discussed in Sect. 2.2, one may argue that Self-Duality can be related to FC(ii), and we considered FC explications that appealed to Self-Duality. However, the FC Explication Requirement rules out such appeal. Prima facie though, there seems to be some leeway to argue that Self-Duality is a punctual property. For don't we say, informally, that Self-Duality requires that, in every claims problem, awards and losses are to be treated symmetrically? And don't we say, formally, that Self-Duality requires that in every claims problem, applying a division rule $r$ and its dual rule $r^{\star}$ result in the same allocation? The problem with such informal paraphrasing of Self-Duality is that it is too imprecise to be helpful. For what does it mean to treat awards and losses symmetrically? To say that it means that $r$ and $r^{\star}$ must coincide in every claims problem serves as a legitimate answer to that question. However, that answer does not allow one to conclude that Self-Duality is a punctual property. Indeed, the application of $r^{\star}$ to $(E, N, c)$ is defined in terms of the application of $r$ to $(L, N, c)$, which is another claims problem that is related to $(E, N, c)$. The following definition of Self-Duality lays bare its relational nature.

Definition 3 (Self-Duality) Let $(E, N, c)$ and $\left(E^{\prime}, N^{\prime}, c^{\prime}\right)$ be claims problems that are related as follows:

$$
E^{\prime}=\sum_{i \in N} c_{i}-E, \quad N^{\prime}=N, \quad c^{\prime}=c
$$

A division rule $r$ satisfies Self-Duality iff for any two claims problems that respect (1), we have that

$$
r(E, N, c)=c-r\left(E^{\prime}, N^{\prime}, c^{\prime}\right)
$$

Definition 3 shows that Self-Duality is a relational property: if claims problems are related as in (1) — the application condition of Self-Duality - then a division rule must, in order satisfy Self-Duality, treat them as in (2) - the treatment condition of Self-Duality. Being a relational property, the FC Explication Requirement precludes Self-Duality from figuring in an FC explication. Of course, the same goes for the other relational property that figured in Theorem 1: Composition ${ }^{6}$.

We investigated, by invoking the axiomatic approach, whether FC can be explicated in terms of properties of division rules and whether we could come up with an explication of FC that characterizes (or implies) P. We started out by considering a particular characterization of P due to Young (1988), viz. Theorem 1. As the substance of three of the four properties appealed to in this characterization seems not be too far removed from FC, Theorem 1 seems to be an attractive starting point for

\footnotetext{
6 In fact, Composition is an example of what Thomson (2012) calls a post-application relational property, whereas Self-Duality is a it pre-application relational property. For sake of completeness, the appendix explains this interesting difference between pre- and post-application relational properties in terms of SelfDuality and Composition.
} 
"FC explication investigations" such as ours. Although Young's characterization of $\mathrm{P}$ is attractive in this sense, it must be noted that several characterizations of $\mathrm{P}$ have been given in the axiomatic literature. We feel that, of these characterizations, the one by Young is closest in spirit to FC. More importantly though, to the best of our knowledge, all characterizations of $\mathrm{P}$ that have been proposed in the literature exploit relational properties. Thus, no such characterization can, according to the FC Explication Requirement, serve as an adequate explication of FC. That is not to say that it is (logically) impossible to characterize $\mathrm{P}$ in terms of punctual properties only. Such is illustrated by the Trivial Proportionality property, which is defined as follows.

Definition 4 (Trivial Proportionality) A division rule $r$ satisfies Trivial Proportionality just in case, in every claims problem $(E, N, c)$ :

$$
r_{i}(E, N, c)=\frac{c_{i}}{\sum_{j \in N} c_{j}} \cdot E \quad \text { for each } i \in N
$$

Indeed, Trivial Proportionality is a punctual property and $\mathrm{P}$ is the unique division rule that satisfies Trivial Proportionality. However, Trivial Proportionality can only be said to characterize $\mathrm{P}$ in a trivial and uninformative manner.

We think that our discussion in this section warrants the following conclusion: there cannot be a non-trivial axiomatic explication of FC that yields $\mathrm{P}$ in the above sense, since it will rely on axiomatic properties of a type that clearly go beyond FC.

\section{Interlude: methods for fairness}

Let us reflect on the preceding explorations of the relation between FC and $\mathrm{P}$ a bit more generally. In Sect. 2.1 we showed that many division rules (such as CEA and CEL, and arguably many others) realise the fairness principle FC. Crucially, the axiomatic approach does not play a role in arguing this. In Sect. 2.2, we then invoked the axiomatic approach to investigate in what way FC can be explicated, and whether such an explication yields $\mathrm{P}$ as the only fair division rule. We concluded Sect. 2.2 negatively. In Sect. 2.3, we offered some thoughts on the underlying reasons for this, by pointing to the distinction between punctual and relational properties.

Where do these findings leave us? While our investigations have yielded a negative conclusion for Curtis' specific claim, we do think that much of what we have offered has been constructive and actually results in a profound challenge to the fairness literature, in particular to Broomean theories, according to which proportional satisfaction of claims is the hallmark of fairness: can the intuitive connection between fairness and proportionality be accounted for on more fundamental grounds? Curtis has made an important start in investigating and answering this question. The axiomatic approach was helpful in showing that there are problems with his answer, and provides the necessary tools to make debates about this challenge precise.

The more general methodological message from applying the axiomatic approach to Curtis' theory of fairness is that any such theory needs to be more explicit in providing a conception of fairness, a specific method that realises it in concrete cases, and a clear story of how the two are connected. We think that the axiomatic approach 
will be a valuable tool to do so for anyone invested in theorising about fair division problems. We will, however, not go on to offer our own theory of fairness based on the axiomatic investigations in this paper. There are three reasons for this: firstly, while we have already included a fair amount of detail of some of the formal properties discussed in the literature, these were primarily geared towards investigating Curtis' claim, and there are many others that will require considerations for any such more general project. It is thus wise to leave that for elsewhere. Secondly, recall that we also mentioned the cooperative game-theoretic literature in the introduction of the paper. It is our view that the results in that literature also deserve consideration and inclusion in an effort to provide a theory of fairness that satisfy the desiderata mentioned here. We thus want to spend the remainder of the paper to demonstrate the relevance of that literature as well. Indeed, there are important challenges to the proportional rule from cooperative game theory ahead. Thirdly and lastly, the Broomean literature on fairness also debates questions concerning the underlying notion of what a claim is, and we will go on to show that these point directly to the game-theoretic methods.

\section{More fairness}

\subsection{Beyond simple claims}

Curtis might also try to defend his theory in a different way: he might try to further spell out his, by and large tacit, assumption of what it means to have a claim, which so far we have simply taken at face value. Curtis actually did the same, but also mentions that he takes his 'cue from Broome's theory of fairness' (Curtis 2014, p. 47). Indeed, Curtis expresses claims in Broomean terms: that is, claims are a perfect record of what agents deserve, they are a duty to the agent (Broome 1990, p. 92f.). Broome is noncommittal as to how such claims are constituted. They may arise due to, for instance, desert, needs, or agreement. For Broome, a claim is thus a specific type of reason why an agent should get the good: a reason that is owed to the agent herself. Claims are to be distinguished from teleological reasons and from side-constraints, where the latter are reasons that necessarily prevail and determine directly what ought to be done.

If we adopt the 'claims as duties'-definition for Bankruptcy, then P seems to have some advantages over CEA and CEL as the latter two rules have some re-distributive properties (this will be intuitively plausible when consulting the table that showed how the three rules divide the estate in Bankruptcy). In less idealized circumstances, CEA and CEL might capture fairness better. Think for instance about an allocation situation quite similar to Bankruptcy in terms of the numbers, but in which the good and claims in question do not reflect Broomean duties. For instance, think of the numbers as representing resources that different agents require to survive. It may well be the case that there is a minimal threshold of resources below which it is impossible for an agent to survive. Then it might be more natural to adopt CEA, which equalizes the awards as much as possible, ensuring a minimal threshold is passed. Alternatively, think of the numbers as representing investments in risky financial products. Then it might be more natural to adopt CEL, which equalizes the losses. All this does help Curtis only to some extent: even when adopting a Broomean definition of a claim, we would still 
need an explicit argument for why P would follow from FC as the only fair division rule.

Broome's (1990) account of fairness has been criticized on various grounds that are connected to his notion of a claim being overly idealized and restrictive (Hooker 2005; Tomlin 2012; Kirkpatrick and Eastwood 2015). For instance, Hooker (2005) argues that, pace Broome, fairness is not insensitive to side-constraints. Another criticism is due to Tomlin (2012), who argues that claims can be (un-)fair by themselves and that Broome cannot account for this feature given that he understands fairness as consisting (solely) of the proportional satisfaction of claims. Thus, Hooker challenges Broome's view that fairness is only concerned with claims, whereas Tomlin challenges Broome's view that claims are logically prior to fairness. The restrictions inherent in Broome's notion of claims and the criticisms voiced by Hooker (2005) and Tomlin (2012) suggest that a theory of fairness, in order to be applicable to a broader set of important cases, should not be bound to a specific idea of claims as what is owed to the agent.

The question thus arises: How can we be fair when we cannot describe claims in terms of Broomean duties, but merely as aspirations or preferences of agents, or as their contribution levels, or else? And what about situations in which we do not really know what claims there are, for instance because that information is not at hand, or it cannot be easily inferred from the context? Can we also consider fairness in such situations?

In order to illustrate these questions, consider the following situation.

Gloves. $A(\mathrm{nn})$ owns 1 left glove and 2 right gloves, $B$ (ernie) own 3 left gloves and 1 right glove and $C$ (harlie) owns 0 left gloves and 1 right glove. At the market, pairs of gloves can be sold for 1 Euro and each pair that is offered for sale is actually sold. The agents can go to he market individually, in pairs, or all three of them together. How should the revenue be divided?

When the agents enter the market as individuals, they will each sell the pairs of gloves that they own. By doing so, Ann and Bernie will earn 1 Euro, and Charlie won't earn anything. However, when the three agents join forces and enter the market together, they can pool their gloves, form 4 pairs and thus jointly earn 4 Euros. As the sum of the individual earnings is only 2 , the three agents have a clear incentive to join forces. And when they do so, the question arises how, in order to be fair, the 4 Euros must be divided amongst the three agents. Here is a comprehensive list of the earnings $v$ for all different combinations of cooperating agents:

$$
\begin{aligned}
& v(\emptyset)=0 \quad v(\{A\})=1 \quad v(\{B\})=1 \quad v(\{C\})=0 \\
& v(\{A, B\})=3 \quad v(\{A, C\})=1 \quad v(\{B, C\})=2 \quad v(\{A, B, C\})=4
\end{aligned}
$$

In some ways, Gloves resembles the fair division problems considered by Curtis. There is however one important exception: the agents are not endowed with claims. We do know the potential revenue they get from cooperating in various constellations, but we do not know which duties we owe to the agents.

As there are different options for each agent to cooperate with other agents, and as the revenue that can be gained from such cooperations varies, agents have different incentives to cooperate. To see this aspect more clearly, consider the following table, 
Table 2 Marginal contributions in gloves

\begin{tabular}{llll}
\hline Order & $M C_{A}$ & $M C_{B}$ & $M C_{C}$ \\
\hline$A, B, C$ & 1 & 2 & 1 \\
$A, C, B$ & 1 & 3 & 0 \\
$B, A, C$ & 2 & 1 & 1 \\
$B, C, A$ & 2 & 1 & 1 \\
$C, A, B$ & 1 & 3 & 0 \\
$C, B, A$ & 2 & 2 & 0 \\
Sum $M C_{i}{ }^{\prime}$ s & 9 & 12 & 3 \\
\hline
\end{tabular}

which lists the 'marginal contribution' (MC) of each agent in all possible cooperation scenarios, as well as the sum of them.

The table can be understood as follows. Consider the row which describes the scenario in which the agents cooperate in the order A,C,B. First, $A$ is alone and receives $v(\{A\})=1$. Then $C$ enters, the two players form a coalition and $C$ receives his marginal contribution of $v(\{A, C\})-v(\{A\})=1-1=0$. Finally, $B$ enters, the three players now present form the 'grand coalition' of all players and $B$ receives his marginal contribution of $v(\{A, B, C\})-v(\{A, C\})=4-1=3$. There are thus clearly differences in the marginal difference that the agents make in the various settings.

The problem is this. Clearly, we do have intuitions that there will be allocations of revenue that are more fair than others. Yet, neither Broome's nor Curtis' theory can tell us what an allocating agent must do in Gloves in order to be fair. In the following, we will introduce the cooperative game theory approach that provides tools to investigate Gloves and similar cases. In Sect. 4.2, we will introduce solution values in cooperative game theory. In Sect. 4.3, we will invoke cooperative game-theory to spell out and argue for the following two claims: (i) unlike other division rules, P lacks a certain kind of robustness, (ii) it is not possible to understand the Gloves situation as being induced by a claims problem, which blocks the potential Broomean rejoinder that fairness is fundamentally a function of claims.

\subsection{Fairness beyond simple claims: using cooperative game theory ${ }^{\star}$}

Gloves as given by (3) is the cooperative game induced by Gloves; let's call it the Gloves Game. More generally, a cooperative game is defined as follows.

Definition 5 A cooperative game is a pair $(N, v)$, with $N=\{1, \ldots, n\}$ a set of agents and with $v: 2^{N} \rightarrow \mathbb{R}_{+}, v(\emptyset)=0$ the characteristic function of the game which specifies the value that each group of cooperating agents (or coalition) can guarantee itself. In particular, $v(N)$ represents the value that the grand coalition $N$ can guarantee itself.

Whereas division rules apply to claims problems, solution values apply to cooperative games.

Definition 6 A solution value $S$ is a function that maps each cooperative game $(N, v)$ to an allocation vector $x \in \mathbb{R}_{+}^{N}$ with the property that $\sum_{i \in N} x_{i} \leq v(N)$. 
Typically, a solution value satisfies Efficiency which means, unsurprisingly, that in each cooperative game, it allocates all of $v(N)$. Another property that is common to (virtually) all solution values that have been proposed in the literature is that of Individual Rationality, according to which the value that is allotted to agent $i$ should always be at least $v(\{i\})$, i.e. the value that she can guarantee by herself. Various solution values have been proposed in the literature and all of them can and typically are understood as proposals to divide the value of the grand coalition "in a fair way" ${ }^{7}$.

Prominent solution values that have been proposed in the literature are the Shapley value (cf. Shapley 1953), the nucleolus (cf. Schmeidler 1969), and the $\tau$-value (cf. Tijs 1981). Roughly speaking, the Shapley value proposes that a fair allocation of $v(N)$ consists in each agent receiving an amount that is equal to his or her 'average marginal contribution' with respect to all coalitions in which the agent may occur. (In Gloves, these are $\varphi(v)_{A}=\frac{9}{6}, \varphi(v)_{B}=\frac{12}{6}$, and $\varphi(v)_{C}=\frac{3}{6}$, which are obtained by dividing the last line of Table 2 for each agent with the number of possible 'cooperation orders', which is 6.) The nucleolus proposes that a fair allocation of $v(N)$ is given by that allocation that 'minimizes the maximal complaint that a coalition can have with respect to an allocation'. Although these descriptions of the Shapley value and the nucleolus are informal, they do reveal that the rationale of these solution values does not involve the notion of proportionality. In contrast, for the $\tau$-value, the notion of proportionality does play an important role. We will discuss this value in more detail.

Here is how the $\tau$-value proposes to allocate the 4 Euros in Gloves on the basis of the Gloves Game $v$ as given by (3). First, we compute the marginal contribution that each of the agents makes with respect to the grand coalition. As an example, the marginal contribution of Ann is equal to $v(N)-v(\{B, C\})=4-2=2$. This marginal contribution is interpreted as an upper payoff as certainly, it is the maximum value that Ann can expect to receive in an allocation of $v(N)$. Writing $U_{A}=2$ to denote that the upper payoff of Ann is 2 , we also find that $U_{B}=3$ and that $U_{C}=1$. Likewise, the lower payoff of an agent represents the minimum value that an agent can expect to receive in an allocation of $v(N)$. To obtain the lower payoff of Bernie, we reason as follows. The lower payoff of Bernie is at least 1 , for this much he can obtain by acting alone: $v(\{B\})=1$. On the other hand, Bernie can also propose to form a coalition with Ann (and without Charlie) by offering Ann her upper payoff of 2 Euros. Given that she is offered her upper payoff, Ann will accept this proposal. By forming a coalition, Ann and Bernie jointly realize 3 Euros which means that, when Ann has received her upper payoff, 1 is left for Bernie. So Bernie cannot only guarantee 1 Euro by acting alone but also via the proposal to Ann that we just sketched. Finally, Bernie may also propose to form the grand coalition and offer both Ann and Charlie their upper payoffs, in which case there is $4-2-1=1$ left for him. In all three scenarios, Bernie can guarantee 1 Euro and so his lower payoff, which is the maximum of the amounts he can guarantee in the three scenarios, is also 1 Euro: $L_{B}=1$. Likewise,

\footnotetext{
7 Just like for division rules, there are two ways in which the notion of fairness that is captured by a solution value can be understood. One can do so axiomatically, i.e. in terms of the fairness of more basic properties that jointly characterize a solution value. Or one can do so procedurally, i.e. in terms of the fairness of the procedure that is used by the solution value to determine the division of $v(N)$. In what follows, we will only be concerned with the procedural aspects of solution values.
} 
one finds ${ }^{8}$ that $L_{A}=1$ and $L_{C}=0$. The $\tau$-value is then obtained ${ }^{9}$ as the following compromise between the vector of lower payoffs $L$ and the vector of upper payoffs $U$ :

$$
\tau(N, v)=\lambda \cdot U+(1-\lambda) \cdot L \quad \text { with } \lambda \text { determined by } \sum_{i \in N} \tau_{i}=v(N)
$$

Although (4) clearly reveals in which sense the $\tau$-value is a compromise between the lower payoffs and upper payoffs, the following (equivalent) expression clearly lays bare in which sense proportionality plays a role.

$$
\tau_{i}(N, v)=L_{i}+\frac{\left(U_{i}-L_{i}\right)}{\sum_{j \in N}\left(U_{j}-L_{j}\right)} \cdot\left(v(N)-\sum_{j \in N} L_{j}\right)
$$

And so in Gloves, the $\tau$-value proposes to allot $\frac{3}{2}$ to Ann, 1 to Bernie and $\frac{1}{2}$ to Charlie, as the reader may easily verify in terms of (5). Indeed, for Gloves, the allocations proposed by the $\tau$-value and the Shapley value coincide.

And so, cooperative game theory provides us with means to approach fair division problems present in situations such as Gloves whereas Curtis' and Broome's limited theories of fairness do not have such means. ${ }^{10}$ Of course, Gloves is only a toy example but cooperative game theory can and has been used to analyse fair division problems that arise in a wide variety of situations, such as the division of profits from production or joint ventures. Although cooperative game theory offers quite some solution values by which such a division can be obtained, only the $\tau$-value, as revealed by (5), explicitly appeals to the notion of proportionality. And so, whereas fair division problems such as Gloves are outside the scope of Broomean theories of fairness, they may be inside the scope of a broader theory of fairness that upholds the connection between fairness and proportionality by appealing to the $\tau$-value. However, in order to be successful, such a theory needs a conception of fairness from which such an appeal to the $\tau$-value can be justified. It is clearly beyond the scope of the present paper to offer such a conception.

\subsection{Fairness and proportionality ${ }^{\star}$}

In the previous section, we have seen that cooperative game theory may be of help when we want to be fair in situations that are outside the scope of Curtis' and Broome's

\footnotetext{
${ }^{8}$ So in this case, the lower payoff of each agent $i$ is equal to the value $v(\{i\})$ he realizes by acting alone. In general, this need not be the case.

9 As the reader may observe, the $\tau$-value for a cooperative game $(N, v)$ is only defined when both $L \leq U$ and $\sum_{j \in N} L_{j} \leq v(N) \leq \sum_{j \in N} U_{j}$. Games that satisfy these two conditions are called quasi-balanced. The limitation of the $\tau$-value to quasi-balanced games can be seen as a weakness of the $\tau$-value. But, as Jene and Zelewski (2012, p. 181) point out, 'a weakness would only exist if theoretically interesting or practically relevant instances of [cooperative games] that do not belong to the class of quasi-balanced games were specified. This has not been the case up to now.'

10 The potential rejoinder that fairness is fundamentally a function of (Broomean) claims, as coalitional strength can be understood in terms of claims, will be addressed at the end of Sect. 4.
} 
theories of fairness. Interestingly though, cooperative game theory has also been used to analyse claims problems, i.e. the situations that are inside the scope of these theories. An essential part of such analysis is the construction of the cooperative game induced by a claims problem, which is aptly illustrated via the Bankruptcy example of Sect. 2. Remember that the characteristic function $v$ of a cooperative game represents the value that a coalition can guarantee itself. And so, as Claire had only 200 on her savings account, Ari and Betty can jointly guarantee themselves $v(\{A, B\})=900-200=$ 700. Indeed, Bankruptcy induces the following Bankruptcy Game:

$$
\begin{aligned}
& v(\emptyset)=0 \\
& v(\{A\})=300 \\
& v(\{B\})=0 \\
& v(\{C\})=0 \\
& v(\{A, B\})=700 \\
& v(\{A, C\})=500 \\
& v(\{B, C\})=100 \\
& v(\{A, B, C\})=900
\end{aligned}
$$

More generally, the cooperative game that is induced by a claims problem is defined as follows.

Definition 7 Let $\mathcal{C}=(E, N, c)$ be a claims problem. The claims game $\left(N, v^{\mathcal{C}}\right)$ that is induced by $\mathcal{C}$ is defined as follows:

$$
v^{\mathcal{C}}(S)=\max \left\{0, E-\sum_{i \notin S} c_{i}\right\} \quad \text { for each } S \subseteq N
$$

An inspection of Definition 1 and 7 reveals that, with $\mathcal{C}$ a claims problem and with $v^{\mathcal{C}}$ the claims game induced by $\mathcal{C}$, we have that $v^{\mathcal{C}}(N)=E$, the value of the grand coalition in the game coincides with the estate of the problem. This observation shows that two methods are available to divide the estate in a claims problem $\mathcal{C}$ : directly, by applying one of the division rules discussed in Sect. 2 to $\mathcal{C}$ or indirectly, by applying one of the solution values mentioned in Sect. 4.2 to the claims game $v^{\mathcal{C}}$. A rule $r$ is said to coincide with solution value $S$ when, for each claims problem $\mathcal{C}$, dividing directly by $r$ coincides with dividing indirectly by $S$.

We will call a rule that coincides with a solution value 'robust'. To explain this label, consider Bankruptcy again. One may model Bankruptcy as a claims problem, as we did in Sect. 2. Doing so, the division of the 900 Euro can proceed in terms of one of the division rules that were discussed in Sect. 2, and the methodology of the axiomatic approach becomes available in terms of which the fairness of such division rules can be investigated. On the other hand, one may model Bankruptcy as a cooperative game, as we did above. Indeed, one may model the fact that Ari had 800 on his savings account by endowing him with a claim $c_{A}$ of 800 , or one may model the fact that Betty and Claire jointly have 600 on their saving accounts as indicating that Ari can guarantee himself $v(\{A\})=900-600=300$. When the latter perspective is taken, the division of the 900 Euro can proceed in terms of one of the solution values that were discussed in Sect. 4.2, and the methodology of cooperative game-theory becomes available in terms of which the fairness of such solution values can be investigated. Thus, we have one fair division situation that can be modelled in two different ways. Say that one has a (non-Broomean) theory of fairness that allows one to asses the fairness of both division rules and of solution values - and note that the need for such 
a theory is illustrated by situations such as Gloves. Arguably, a desirable feature of such a theory of fairness is that the allocation of the 900 Euros in Bankruptcy (and similar situations) does not depend on the manner in which Bankruptcy is modelled. That is to say, it is a desirable feature of such a theory of fairness that its preferred division rule coincides with its preferred solution value. For instance, the 900 Euros in Bankruptcy and the 4 Euros in Gloves can then be divided according to the very same principles. A necessary condition for the realization of this desirable feature is that the division rule $r$ that is proposed by the theory is robust: there should at least be $a$ solution value that coincides with $r$.

An interesting example of a robust rule is the Talmud rule (T) that was (implicitly) proposed as a fair division rule in the Talmud. Around 2000 years later, Schmeidler (1969) proposed the nucleolus as a fair solution value for cooperative games, and Aumann and Maschler (1985) showed that T coincides with the nucleolus, and so $\mathrm{T}$ is robust in our sense. Although many division rules are robust ${ }^{11}$, the proportional rule $\mathrm{P}$ is not one of them, as the following proposition attests.

Proposition 3 With $\mathcal{C}=(E, N, c)$ a claims problem, let $\overline{\mathcal{C}}=(E, N, \bar{c})$ be the claims problem that is defined just like $\mathcal{C}$ except that all claims are truncated to $E$, i.e. $\bar{c}_{i}=\min \left\{E, c_{i}\right\}$ for each agent $i$. There is a a solution value $S$ such that $r$ coincides with $S$ if and only if, for each claims problem $\mathcal{C}$, applying $r$ to $\mathcal{C}$ yields the same division as applying $r$ to $\overline{\mathcal{C}}$.

\section{Proof Curiel et al. (1988).}

An immediate consequence of Proposition 3 is that $P$ is not robust. Now, from the perspective of Curtis (or Broome), one may not be too worried about this result. For Proposition 3 essentially tells us that the only way in which a division rule can be robust is by considering the part of a claim that exceeds the estate-if there is such part-as irrelevant. There is nothing in the Broomean notion of a claim-a duty we owe to the agent - that justifies this attitude towards estate-exceeding claims. Yet, we (and others) already argued that not all fair division problems are cast in terms of Broomean claims. At the end of Sect. 4.2, we already hinted at a theory of fairness that gives up the Broomean notion of a claim but that keeps a connection between fairness and proportionality. We argued that for such a theory, the $\tau$-value may be an appealing solution value. Interestingly, the Adjusted Proportional rule is a robust division rule that coincides with the $\tau$-value.

Theorem 2 Let $\mathcal{C}=(E, N, c)$ be an arbitrary claims problem and let $v^{\mathcal{C}}$ be the claims game that is induced by $\mathcal{C}$. Applying the $A P$ rule to $\mathcal{C}$ results in the same allocation as applying the $\tau$-value to $v^{\mathcal{C}}$.

Proof Curiel et al. (1988).

And so, for a non-Broomean theory of fairness that wants to keep a connection between fairness and proportionality, the AP rule seems to be an attractive division rule.

\footnotetext{
11 As another example, the Run to the Bank rule, mentioned in Sect. 2.2, coincides with the Shapley value and hence, it is robust. For yet another example, see Theorem 2 below.
} 
We want to conclude this section by addressing a potential objection to an argument made earlier. We pointed out that a fair division of the 4 Euros in Gloves can be obtained by applying a solution value to the Gloves Game. Hence, a fair division of the 4 Euros is obtained as a function of coalitional strength, that is, of how much leverage each agent has in terms of forming beneficial coalitions. The thought that, in Gloves, fairness is a function of coalitional strength is in conflict with the Broomean thought that fairness is always a function of claims. Gloves (which clearly gives rise to a fair division problem) thereby seems to discredit the Broomean account of fairness. However, Broome and Curtis may argue that fairness is fundamentally a function of (Broomean) claims, as coalitional strength can be understood in terms of claims. That is, they may argue that the structure of the Gloves situation as given by the Gloves game is induced by a more fundamental structure that can be spelled out in terms of claims. For instance, they could argue that the fact that Ann can guarantee herself 1 Euro can be explained in terms of (implicit) claims on the 4 Euros that can be ascribed to Bernie and Charlie. The following proposition attests that this kind of argument is impossible.

Proposition 4 Let $v$ be the Gloves Game as given by (3). For no claims problem $\mathcal{C}$ we have that $v^{\mathcal{C}}=v$.

Proof Let $v$ be the Gloves Game and suppose that there is a claims problem $\mathcal{C}=$ $(E,\{A, B, C\}, c)$ such that $v^{\mathcal{C}}=v$. Then, as $v^{\mathcal{C}}(\{A, B, C\})=4$, the estate $E$ should equal 4. Further, as $v^{\mathcal{C}}(\{A, B\})=3$, it follows that Charlie's claim $c_{C}=1$. Likewise, one derives that $c_{A}=2$ and that $c_{B}=3$. But then $v^{\mathcal{C}}(\{A\})=4-3-1=0=$ $v(\{A\})=1$ which is absurd.

Hence, simply pointing out that any context in which we may want to say something about fairness should be re-described as an allocation situation with Broomean claims won't work: the latter cannot always be constructed on the basis of fair division problems such as Gloves. Theorising about fair division problems will thus be severely restricted if it is limited to those in which highly idealised claims Broomean claims are given. We will elaborate on this restriction a bit further in the next section.

\section{Coda: fair division problems, allocative justice, distributive justice}

Let us again reflect more generally about the preceding explorations. We have considered the challenge, issued by Hooker (2005) and Tomlin (2012), against the highly restrictive notion of Broomean claims, which have been employed in Curtis' (2014) theory of fairness. In Sect. 4.2, we showed that cooperative game theory is a framework that allows us to explore notions of fair divisions in contexts in which no Broomean claims are given. In Sect. 4.3, we then showed that many division rules coincide with a solution value of cooperative game-theory, but not the proportional rule. We also showed that there is a class of fair division problems in which Broomean claims cannot be constructed.

Where do these suggestions leave us? We think it important to appreciate that the problems considered by Broomean theories of fairness, and by the axiomatic and game-theoretic approaches used in our article, are somewhat different from more 
fundamental considerations covered by theories of distributive justice. At the same time, it is not quite clear in what respect these problems are different exactly, and how to distinguish between fair division problems and those of distributive justice. Consider this passage from Rawls, in which he introduces one way of distinguishing allocative and distributive justice:

By contrast [to distributive justice] allocative justice applies when a given collection of goods is to be divided among definite individuals with known desires and needs. The goods to be allotted are not produced by these individuals, nor do these individuals stand in any existing cooperative relation. Since there are no prior claims on the things to be distributed, it is natural to share them out according to desires and needs, or even to maximize the net balance of satisfaction. Justice becomes a kind of efficiency, unless equality is preferred. Suitably generalized, the allocative conception leads to the classical utilitarian view.' (Rawls 1971, p. 77)

There are many ways in which this quote describes allocative justice that corresponds closely to the kind of fair division problems that we have analysed in this article. For instance, in both Bankruptcy and Gloves, there are fixed amounts of goods to be distributed, and agents have claims, preferences, or contributions that need to be recognized when one wants 'to be fair'. At the same time, there is clearly something about these cases that does not make them straightforward to treat by simple utility maximisation.

How does this passage from Rawls relate to the theories that we covered in this article? Reconciling the notion of Broomean claims with this passage from Rawls is difficult. On the one hand, Broomean claims seem to go together quite well with Rawls stating that there is no cooperation or production. Likewise, Rawls also seems close to the idea of efficiency as expressed in Curtis (2014) FC(i). And it seems to us that in general, the spirit in which Rawls defines allocative justice is quite similar to what Broomean theories of fairness attempt to cover. On the other hand, Rawls does seem to assume that allocative justice deals with the currency of utility, rather than agents' entitlements as Broome does. Reconciling the axiomatic and cooperative game theory literature with this passage from Rawls is also not straightforward. As we have seen, cooperative game theory applies to cases in which there is cooperation and production, contrary to the above. Likewise, axiomatic approaches and cooperative game theory are not bound to one currency of utility (although most work done in these approaches assume it).

Interestingly, while the examples of Bankruptcy and Gloves proved to be poignant ways in which to explore relations between Broomean theories of fairness and the economic literature, the picture looks much more complex when it comes to define the field in which these theories operate: there is no straightforward correspondence between Rawls, Broome, and the authors that responded to the latter and the economic literature. We highlight this fact for several reasons: firstly, we think this might partly explain why even though there is a long tradition in theories of allocative and distributive justice to integrate philosophy and economics (such as in the work of Gauthier (1986), Roemer (1998), Fleurbaey (2008), and Fleurbaey and Maniquet (2011), to name only a few), Broomean theories of fairness have so far proceeded in isolation 
from the economic literature. Secondly, we also think that our discussion suggests that it is an open question whether there is in fact a clearcut distinction between fair division, allocative justice, and distributive justice: there might be interesting relations between methods used in either area.

Neither do we wish to defend views on fundamental questions such as whether and how to distinguish between fair division, allocative justice, and distributive justice, nor do we want to put allocative justice as the definite label for the kinds of problems we have considered here. We do however think that theorising about fair division problems will benefit from clarifying such matters in the future. Our contribution in this article is confined to issue the specific challenges to Curtis and Broome, as developed in Sects. 2 and 4, as well as to generally suggest that the methods of axiomatic and game-theoretic approaches should not be ignored when thinking about fair division problems.

\section{Conclusions}

We have introduced axiomatic and game theoretic approaches from economics to confront current philosophical debates on fair division problems. Confronting the philosophical literature with these methods yields a number of interesting results. Firstly, we show that the proportionality method advocated in Curtis (2014) is not implied by a general principle of fairness, and that the proportional rule cannot be explicated axiomatically from that very principle. Secondly, we show that adopting Broome's (1990) restricted definition of the scope of theorising about fair division problems excludes some of the most important such problems in which one would like to analyse fairness, and that game-theoretic approaches can rectify this shortcoming. We thus demonstrate that using axiomatic and game-theoretic work in economics can rectify shortcomings in the philosophical literature on fair division problems, and argue that it is an indispensable ingredient of any theorizing about fair division, allocative justice, and distributive justice.

Acknowledgements Many thanks to Sine Bağatur, Constanze Binder, F.A. Muller, Alice Obrecht, audiences at Erasmus University Rotterdam and the European Philosophy of Science Association (EPSA) Biennial Conference 2015, as well as referees for Synthese. Stefan Wintein thanks the Netherlands Organisation for Scientific Research (NWO) for funding the project The Structure of Reality and the Reality of Structure (project leader: F.A. Muller), in which he is employed. Conrad Heilmann's work has been supported by a Marie Curie Career Integration Grant from the European Union and a VENI grant from the Netherlands Organisation for Scientific Research (NWO).

Open Access This article is distributed under the terms of the Creative Commons Attribution 4.0 International License (http://creativecommons.org/licenses/by/4.0/), which permits unrestricted use, distribution, and reproduction in any medium, provided you give appropriate credit to the original author(s) and the source, provide a link to the Creative Commons license, and indicate if changes were made.

\section{Appendix}

\section{Proof of Lemma 1}

Lemma 1 Let $(E, N, c)$ be a claims problem and let $k, l \in N$ be such that $c_{k}<c_{l}$. Then $A P_{k}(E, N, c)<A P_{l}(E, N, c) \Longleftrightarrow c_{k}<E$. 
Proof Let us first present the formal definition of the AP rule in order to facilitate our proof. Let $(E, N, c)$ be a claims problem. For each agent $i \in N, m_{i}=\max \{0, E-$ $\left.\sum_{j \neq i} c_{j}\right\}$ denotes the minimal right of agent $i$. We use $E^{R}=E-\sum_{i \in N} m_{i}$ to denote the remaining estate and we leave it to the reader to observe that the definition of a claims problem implies that $E^{R}>0$. Further, we use $c_{i}^{R}=\min \left\{E^{R}, c_{i}-m_{i}\right\}$ to denote the remaining claim of agent $i$ and we let $c^{R}$ denote the reduced claims vector thus obtained. The amount allotted to agent $i$ by the AP rule may thus be expressed as

$$
A P_{i}(E, N, c)=m_{i}+P_{i}\left(E^{R}, N, c^{R}\right)
$$

Left-to-right direction. Reason by contraposition. Let $k, l \in N$ be such that $c_{k}<c_{l}$ and suppose that $c_{k} \geq E$. Then also $c_{l} \geq E$. It readily follows that $m_{k}=m_{l}=0$ and that $c_{k}^{R}=c_{l}^{R}=E^{R}$. As P satisfies Equal Treatment of Equals it readily follows from (7) that $A P_{k}(E, N, c)=A P_{l}(E, N, c)$, which establishes the desired result.

Right-to-left direction. Let us first find a convenient expression for the remaining estate $E^{R}$. To do so, let $C=\sum_{i \in N} c_{i}$ and $L=C-E$. We than have that $m_{i}=$ $\max \left\{0, E-\sum_{j \neq i} c_{j}\right\}=\max \left\{0, E-C+c_{i}\right\}=\max \left\{0, c_{i}-L\right\}$. Now let $S=\{i \in$ $\left.N \mid m_{i}>0\right\}$ and let $T=N-S$. We have:

$$
E^{R}=E-\sum_{i \in S}\left(c_{i}-L\right)+\sum_{i \in T} c_{i}-\sum_{i \in T} c_{i}=(|S|-1) \cdot L+\sum_{i \in T} c_{i}
$$

Now let $k, l \in N$ be such that $c_{k}<c_{l}$ and suppose that $c_{k}<E$. Suppose that $S=\emptyset$. Then $m_{k}=m_{l}=0$ and $E^{R}=E$. And so $c_{k}^{R}=\min \left\{E, c_{k}\right\}=c_{k}$ and $c_{l}^{R}=\min \left\{E, c_{l}\right\}>c_{k}$. As $m_{k}=m_{l}=0$ and as P satisfies Strict Order Preservation, it follows from (7) that $A P_{k}(E, N, c)<A P_{l}(E, N, c)$. For the remainder of the proof, we may thus suppose that $S \neq \emptyset$.

Suppose that $m_{l}=0$. As $c_{k}<c_{l}$, it follows that $m_{k} \leq m_{l}$ and hence that $m_{k}=0$. As $S \neq \varnothing$ and as $l, k \in T$, it follows from (8) that $c_{k}^{R}=c_{k}$ and that $c_{l}^{R}=c_{l}$. Again, the desired result follows from the fact that $\mathrm{P}$ satisfies Strict Order Preservation. Now suppose that $m_{l}>0$ and that $m_{k}=0$. As $S \neq \varnothing$ and as $k \in T$, it follows from (8) that $c_{k}^{R}=\min \left\{E^{R}, c_{k}\right\}=c_{k}$. As $m_{l}>0$, it follows that $m_{l}=c_{l}-L$ so that $c_{l}^{R}=\min \left\{E^{R}, L\right\}$. As $m_{k}=0$, it follows that $c_{k}-L \leq 0$ and so $c_{k} \leq L$. Thus $c_{k}^{R}=c_{k} \leq \min \left\{E^{R}, L\right\}=c_{l}^{R}$. As $m_{l}>m_{k}$ and as $\mathrm{P}$ satisfies Equal Treatment of Equals and Strict Order Preservation, the desired result follows from (7). Finally, suppose that $m_{l}>0$ and that $m_{k}>0$. It follows that $c_{k}^{R}=c_{l}^{R}=\min \left\{E^{R}, L\right\}$ and, as $c_{k}<c_{l}$, that $m_{k}<m_{l}$. Hence, as $\mathrm{P}$ satisfies Equal Treatment of Equals, the desired result follows from (7).

\section{Proof of Proposition 1}

Proposition 2 P does and CP does not satisfy Strict Claim Monotonicity.

Proof Let us first present the formal definition of Strict Claim Monotonicity in order to facilitate our proof. Let $\mathcal{C}=(E, N, c)$ and $\mathcal{C}^{\prime}=\left(E, N, c^{\prime}\right)$ be two claim problems 
with the same estate $E$ and the same set of agents $N$ and with claims vectors $c$ and $c^{\prime}$ such that:

$$
\left(\exists i \in N: c_{i}<c_{i}^{\prime}\right) \text { and }\left(\forall j \in N: j \neq i \Longrightarrow c_{j}=c_{j}^{\prime}\right)
$$

A division rule $r$ satisfies Strict Claim Monotonicity just in case $r_{i}(E, N, c)<$ $r_{i}\left(E, N, c^{\prime}\right)$.

$\mathrm{P}$ satisfies Strict Claim Monotonicity. Let $\mathcal{C}$ and $\mathcal{C}^{\prime}$ be as indicated above and let $C=\sum_{i \in N} c_{i}$ and $C^{\prime}=\sum_{i \in N} c_{i}^{\prime}$ the sums of claims in, respectively, $\mathcal{C}$ and $\mathcal{C}^{\prime}$. Let $j \neq i \in N$ and observe that, as $C<C^{\prime}$ :

$$
P_{j}(E, N, c)=\frac{c_{j}}{C}>\frac{c_{j}}{C^{\prime}}=P_{j}\left(E, N, c^{\prime}\right)
$$

Thus, (9) tells us that, for each agent $j$ other than $i, \mathrm{P}$ allots more to $j$ in $\mathcal{C}$ than in $\mathcal{C}^{\prime}$. As $\mathrm{P}$ satisfies Efficiency, it readily follows that $\mathrm{P}$ allots more to $i$ in $\mathcal{C}^{\prime}$ than in $\mathcal{C}$, which is what we had to show.

To show that $\mathrm{CP}$ does not satisfy Strict Claim Monotonicity, we give a counterexample. Let $\mathcal{C}=(E, N, c)$ and $\mathcal{C}^{\prime}=\left(E, N, c^{\prime}\right)$ be such that $E=500, N=\{1,2,3\}$, $c_{1}=800, c_{1}^{\prime}=801, c_{2}=c_{2}^{\prime}=400$ and $c_{3}=c_{3}^{\prime}=200$. Observe that $C P_{i}(E, N, c)=A P_{i}(E, N, c)=A P_{i}\left(E, N, c^{\prime}\right)=C P_{i}\left(E, N, c^{\prime}\right)$, and so CP violates Strict Claim Monotonicity.

\section{Pre-application and post-application relational properties}

Whereas Self-Duality is a pre-application relational property, Composition is a postapplication relational property. The following definition of Composition will facilitate our explanation of the difference between pre- and post-application relational properties.

Definition 8 (Composition) Let $r$ be a rule and let $(E, N, c),\left(E^{\prime}, N^{\prime}, c^{\prime}\right)$ and $\left(E^{\prime \prime}, N^{\prime \prime}, c^{\prime \prime}\right)$ be claims problems that are related as follows:

$$
E=E^{\prime}+E^{\prime \prime}, \quad N^{\prime}=N, \quad c^{\prime}=c, \quad c^{\prime \prime}=c-r\left(E^{\prime}, N, c\right)
$$

$r$ satisfies Composition iff for any three claims problems that respect (10), we have that

$$
r(E, N, c)=r\left(E^{\prime}, N^{\prime}, c^{\prime}\right)+r\left(E^{\prime \prime}, N^{\prime \prime}, c^{\prime \prime}\right)
$$

Definition 8 clearly shows that Composition is a relational property: if claims problems are related as in (10) - the application condition of Composition-then a division rule must, in order to satisfy Composition, treat them as in (11) - the treatment condition of Composition. As the application condition of Self-Duality can be specified without applying the division rule that figures in its treatment condition, it is a pre-application relational property. In order to specify the application condition of Composition, however, we need to apply the division rule that figures in its treatment condition, and so Composition is a post-application relational property. 


\section{References}

Aumann, R. J., \& Maschler, M. (1985). Game theoretic analysis of a bankruptcy problem from the Talmud. Journal of Economic Theory, 36, 195-213.

Broome, J. (1990). Fairness. Proceedings of the Aristotelian Society, 91, 87-101.

Curiel, I. J., Maschler, M., \& Tijs, S. H. (1988). Bankruptcy games. Zeitschrift für Operations Research, 31, A143-A159.

Curtis, B. L. (2014). To be fair. Analysis, 74, 47-57.

Fleurbaey, M. (2008). Fairness, responsibility, and welfare. Oxford: Oxford University Press.

Fleurbaey, M., \& Maniquet, F. (2011). A theory of fairness and social welfare. Cambridge: Cambridge University Press.

Gauthier, D. (1986). Morals by agreement. Oxford: Oxford University Press.

Herrero, C., \& Villar, A. (2001). The three Musketeers: four classical solutions to bankruptcy problems. Mathematical Social Sciences, 42, 307-28.

Hooker, B. (2005). Fairness. Ethical Theory and Moral Practice, 8, 329-52.

Jene, S., \& Zelewski, S. (2012). Fair distribution of added values in networks of autonomous actors. In H. Jodlbauer, et al. (Eds.), Modelling value, contributions to management science (pp. 167-186). New York: Springer.

Kirkpatrick, J. R., \& Eastwood, N. (2015). Broome's theory of fairness and the problem of quantifying the strengths of claims. Utilitas, 26, 331-345.

O'Neill, B. (1982). A problem of rights arbitration from the Talmud. Mathematical Social Sciences, 2, $345-71$.

Rawls, J. (1971). A theory of justice. Oxford: Oxford University Press.

Roemer, J. E. (1998). Theories of distributive justice. Harvard: Harvard University Press.

Schmeidler, D. (1969). The nucleolus of a characteristic function game. SIAM Journal of Applied Mathematics, 17(6), 1163-70.

Shapley, L. S. (1953). A value for n-person games. In H. W. Kuhn \& A. W. Tucker (Eds.), Contributions to the theory (pp. 307-317)., Annals of mathematical studies v. 28 Princeton: Princeton University Press.

Thomson, W. (2003). Axiomatic and game-theoretic analysis of bankruptcy and taxation problems: a survey. Mathematical Social Sciences, 45, 249-297.

Thomson, W. (2012). On the axiomatics of resource allocation: interpreting the consistency principle. Economics and Philosophy, 28(3), 385-421.

Tijs, S. H. (1981). Bounds for the core and $\tau$-value. In O. Moeschlin \& D. Pallaschke (Eds.), Game theory and mathematical economics (pp. 123-132). Amsterdam: North-Holland Publ Cie.

Tomlin, P. (2012). On fairness and claims. Utilitas, 24, 200-213.

Young, P. (1988). Distributive justice in taxation. Journal of Economic Theory, 43, 321-335. 